Research report

\title{
Response properties of nociceptive neurons in the caudal ventrolateral medulla (CVLM) in monoarthritic and healthy control rats: Modulation of responses by the paraventricular nucleus of the hypothalamus (PVN)
}

\author{
Filipa Pinto-Ribeiro ${ }^{\mathrm{a}, \mathrm{b}}$, Osei B. Ansah ${ }^{\mathrm{a}}$, Armando Almeida ${ }^{\mathrm{b}}$, Antti Pertovaara ${ }^{\mathrm{a}, *}$ \\ a Institute of Biomedicine/Physiology, POB 63, University of Helsinki, 00014 Helsinki, Finland \\ ${ }^{\mathrm{b}}$ Life and Health Sciences Research Institute (ICVS), School of Health Sciences, University of Minho, Campus de Gualtar, $4710-057$ Braga, Portugal
}

\section{A R T I C L E I N F O}

\section{Article history:}

Received 29 April 2011

Received in revised form 22 June 2011

Accepted 25 June 2011

Available online 1 July 2011

\section{Keywords:}

Caudal ventrolateral medulla

Arthritic pain

Descending modulation

Hypothalamus

\begin{abstract}
A B S T R A C T
We characterized response properties of neurons in the caudal ventrolateral medulla (CVLM), a structure involved in pain modulation. Electrophysiological recordings were performed in pentobarbitoneanesthetized control and monoarthritic rats. Noxious pinch, heat, cold and colorectal distension were used for peripheral test stimulation. To study central modulation of CVLM neurons and role in mediating descending pain regulation from the hypothalamus, glutamate was administered into the paraventricular nucleus of the hypothalamus (PVN). CVLM neurons gave excitatory, inhibitory or no response to noxious test stimulation. Response patterns for part of the neurons varied with submodality of test stimulation; e.g., a cell with an excitatory response to heat could give no or an inhibitory response to cold. Arthritis induced cell type-dependent changes in the spontaneous activity, most prominent of which was increased discharge rate of CVLM cells with an excitatory response to noxious stimulation. Significant arthritis-induced changes were also observed in the magnitudes of the nociceptive responses, among which was an increase in the pinch-induced excitatory response. Glutamate in the PVN of arthritic but not control animals suppressed the excitatory responses of CVLM cells, independent of the submodality of test stimulation and without influence on their spontaneous discharge rates. The results indicate that CVLM neurons give differential responses to noxious stimulation. Arthritis induces changes in response properties of CVLM neurons and in their central regulation by the PVN. These findings are in line with the evidence indicating that the CVLM plays a role in processing of nociceptive signals under inflammatory as well as control conditions.
\end{abstract}

(c) 2011 Elsevier Inc. All rights reserved.

\section{Introduction}

Earlier studies indicate that electrical or glutamatergic stimulation of the caudal ventrolateral medulla (CVLM) suppresses spinal nociception $[11,16,21,25]$. Conversely, lesions or blockade of the CVLM facilitate spinal nociception $[9,13,36]$. On the other hand, CVLM seems to be also involved in pronociception as shown by the hyperalgesia induced by local injection of angiotensin II, an effect that is partially reverted by CVLM injection of angiotensin-1 antagonists $[23,24]$. Together these results suggest that descending pathways originating in or relaying through the CVLM play a role in phasic and tonic control of spinal nociception, and that this area can have an antinociceptive and/or pronociceptive role in nociception. While the CVLM appears to play a role in descending pain mod-

Abbreviations: CVLM, caudal ventrolateral medulla; PVN, paraventricular nucleus of the hypothalamus; RVM, rostroventromedial medulla.

* Corresponding author. Tel.: +358 9191 25280; fax: +358 919125302.

E-mail address: antti.pertovaara@helsinki.fi (A. Pertovaara). ulation, the response properties of pain-related CVLM neurons in healthy animals or animals with chronic pain, however, are still poorly known.

It has been proposed that the CVLM modulation of nociceptive transmission is mediated through direct and indirect pathways to the spinal cord; the modulatory mechanisms appear to be specific to the submodality of noxious stimulation [38]. Also, the CVLM may be involved in mediation of the hypoalgesia induced by increased blood pressure, through a CVLM-A5-spinal cord circuitry [36,37]. In addition, the CVLM and the nucleus tractus solitarius are part of a three-way loop that involves supraspinal reciprocal projections with the paraventricular nucleus of the hypothalamus (PVN) [17,18]. The PVN is a major integration center of several circuits, including the stress response through the hypothalamus-pituitary-adrenal axis [3] and descending pain modulation [e.g. 4,6,33,40]. Direct oxytocinergic projections from the PVN to the spinal cord have been shown to be sufficient for mediating phasic antinociception induced by PVN stimulation [5]. Additionally, our recent results indicated that a relay in the rostroventromedial medulla (RVM) may, at least partly, contribute to the 

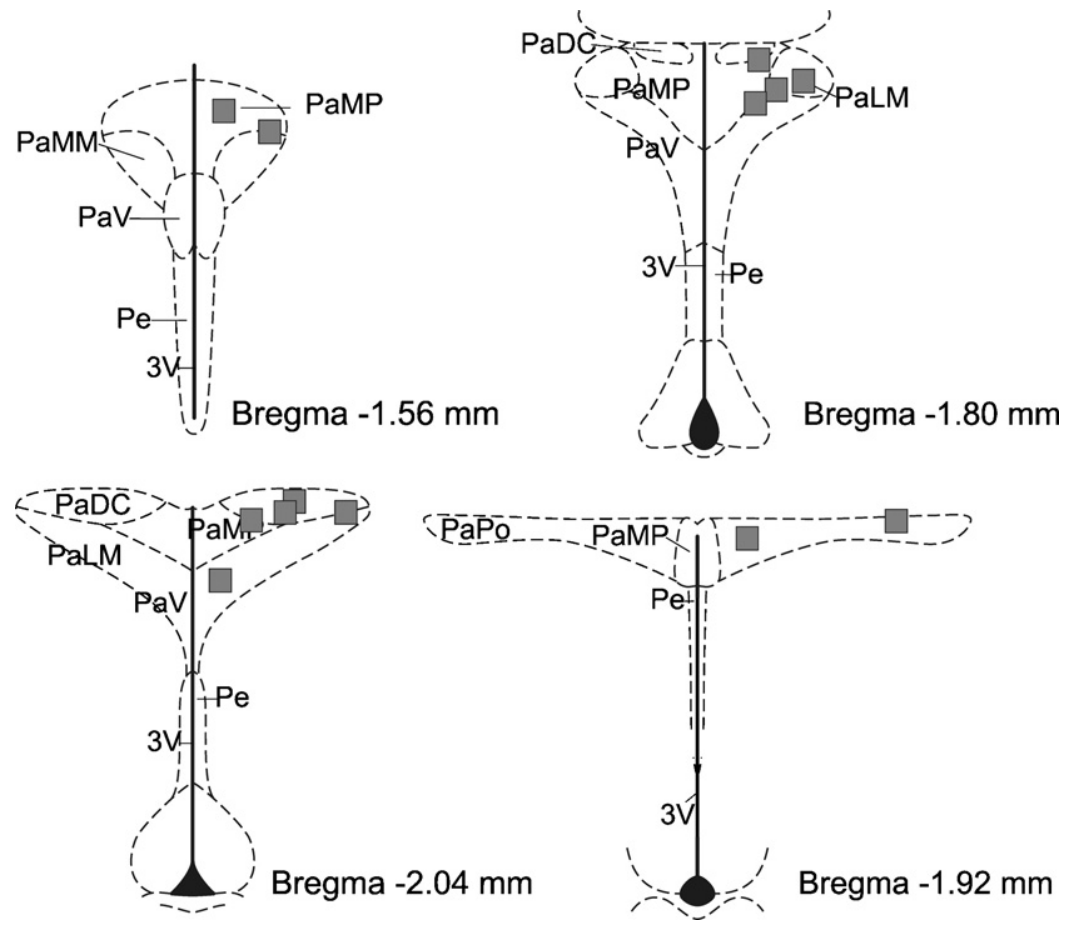

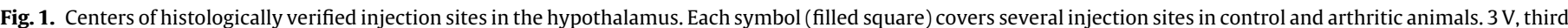

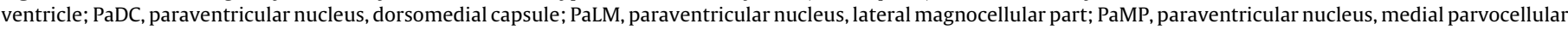
part; PaV, paraventricular nucleus, ventral part; Pe, periventricular nucleus.

mediation of the descending antinociceptive effect from the PVN to the spinal cord in arthritic and healthy control animals [31]. It is not yet known if the PVN modulates pain-related activity in the CVLM or if the CVLM might provide an alternative relay for descending pain modulatory effect from the PVN to the spinal cord level in control or pathophysiological conditions.

Our purpose in this study was to: (i) characterize response properties of nociceptive CVLM neurons using various types of noxious test stimulation in healthy control rats; (ii) analyze potential changes in response properties of CVLM neurons after the induction of monoarthritis; (iii) investigate central regulation of the CVLM by another pain controlling brain site by determining the influence of glutamatergic activation of the PVN on responses of CVLM neurons.

\section{Materials and methods}

\subsection{Experimental animals}

The experiments were performed with 40 adult male Han-Wistar rats with 175-250 g (Harlan Netherlands, Horst, The Netherlands). The experimental protocol was approved by the Ethical Commission of the Regional Government of Southern Finland (Hämeenlinna, Finland) and followed the European Community Council Directive 86/609/EEC for the use of experimental animals. All efforts were made to minimize animal suffering and to use only the number of animals necessary to produce reliable scientific data.

\subsection{Anesthesia}

For the experimental surgery and electrophysiological sessions, anesthesia was induced by administering pentobarbitone $(50 \mathrm{mg} / \mathrm{kg}$, i.p.) and maintained by infusing pentobarbitone $(15-20 \mathrm{mg} / \mathrm{kg} / \mathrm{h})$. If the animal had spontaneous movements, vocalizations or visible reactions to noxious test stimulation, an additional dose of pentobarbitone was administered $(15-20 \mathrm{mg} / \mathrm{kg})$. The level of anesthesia was frequently monitored by observing the size of the pupils, the general muscle tone and behavioural responses to noxious pinching. Importantly, the anesthesia level was maintained in an identical fashion when studying control and arthritic animals to avoid a potential influence of this factor among groups. A warming blanket was used to maintain the body temperature within physiological range.

\subsection{Procedures for intracerebral microinjections}

For glutamate administration into the hypothalamic PVN, the rats were inserted a chronic guide cannula at least one week before the electrophysiological experiment. For installation of the cannula, the anesthetized animal (see above for details of anesthesia) was placed in a stereotaxic frame and a stainless steel guide cannula (26 gauge; Plastics One, Roanoke, VA, USA) was implanted in the brain according to the coordinates of the atlas by Paxinos and Watson [30]. The tip of the guide cannula was positioned $1 \mathrm{~mm}$ above the desired injection site in the PVN (AP, $7.2 \mathrm{~mm}$; LM, $0.2 \mathrm{~mm}$; DV, $7.9 \mathrm{~mm}$ to the interaural line). After the guide cannula was fixed into the skull using a dental screw and dental cement, a dummy cannula was inserted into the guide cannula. Animals were allowed to recover from surgery for at least one week before testing.

Glutamate ( $50 \mathrm{nmol}$; Sigma-Aldrich, St. Louis, MO, USA) was administered into the PVN through a 33-gauge injection cannula (Plastics One) inserted into and protruding $1 \mathrm{~mm}$ beyond the tip of the guide cannula. The microinjection was made using a 1.0- $\mu$ L Hamilton syringe (Hamilton Bonaduz AG, Bonaduz, Switzerland) connected to the injection cannula by a polyethylene catheter (PE-10; Plastics One). The injection volume was $0.5 \mu \mathrm{L}$ and therefore, the spread of the injected drugs within the brain was expected to be at least about $1 \mathrm{~mm}$ [28]. The efficacy of injection was monitored by watching the movement of a small air bubble through the tubing. The injection lasted $20 \mathrm{~s}$ and the injection cannula was left in place for an additional $30 \mathrm{~s}$ to minimize the return of drug solution back to the injection cannula. Brain injection sites were histologically verified from post-mortem sections and plotted on standardized sections derived from the stereotaxic atlas of Paxinos and Watson ([30] Fig. 1). It should be noted that glutamate was microinjected into the PVN at a site and dose that has proved effective in producing spinal antinociception in arthritic as well as healthy control rats [31].

\subsection{Recording of neuronal responses in the caudal ventrolateral medulla (CVLM)}

Recordings of single CVLM neurons were performed under pentobarbitoneinduced anesthesia $\geq 1$ week after installation of the chronic guide cannula into the PVN (see above) and 1-2 weeks after induction of arthritis (see below). After induction of anesthesia (see above), the animals were placed in a standard stereotaxic frame. The skull was exposed and a hole was drilled for placement of a recording electrode in the CVLM ( $4.08 \mathrm{~mm}$ posterior to the interaural line, $2.0-2.2 \mathrm{~mm}$ lateral from the midline, and $10.0 \mathrm{~mm}$ ventral from the dura mater) according to the atlas of Paxinos and Watson [30] (Fig. 2). The precise limits of the CVLM are not clearly defined. The CVLM is commonly described as an area of the medullary reticular formation lateral to the lateral reticular nucleus and medial to the ventral tip of the spinal trigeminal nucleus [20]. Single neuron activity was recorded extracellularly with lacquer-coated tungsten electrodes (tip impedance 3-10 M $\Omega$ at $1 \mathrm{kHz}$; FHC Inc., Bowdoinham, ME, USA) and then amplified and filtered using standard techniques. 

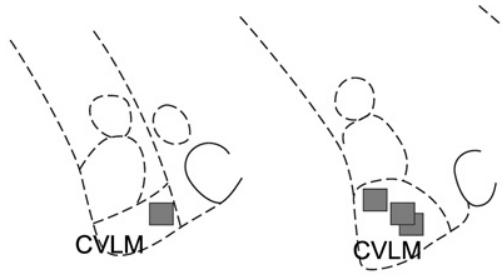

Bregma $-12.60 \mathrm{~mm}$

Bregma $-12.72 \mathrm{~mm}$

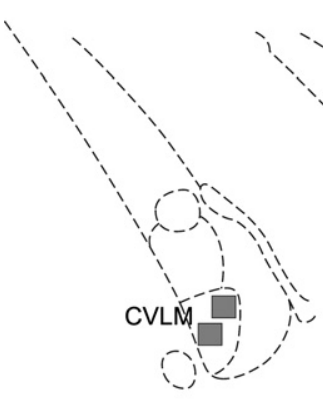

Bregma $-13.08 \mathrm{~mm}$

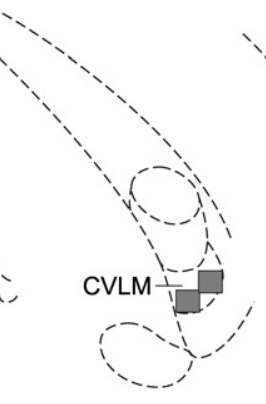

Bregma $-13.32 \mathrm{~mm}$

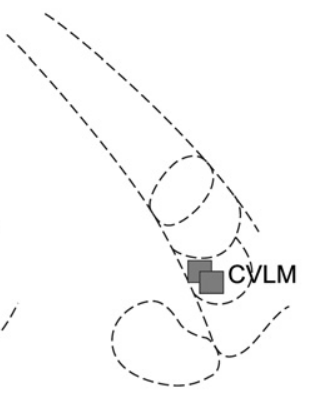

Bregma $-13.44 \mathrm{~mm}$
Fig. 2. Histologically verified recording sites in the caudal ventrolateral medulla (CVLM). Each symbol (filled square) covers several recording sites in control and arthritic animals.

Data sampling was performed with a computer connected to a CED Micro 1401 interface and using Spike 2 software (Cambridge Electronic Design, Cambridge, U.K.).

Recording of CVLM neurons started after the animal was under light anesthesia; i.e., the animals gave a brief withdrawal response to noxious pinch, but the pinch did not produce any longer lasting motor activity, nor did the animals have spontaneous limb movements. CVLM neurons were classified based on their response to noxious test stimulation consisting of heat, pinch, colorectal distension and cold (see below).

A modified version of the classification scheme developed earlier for RVM neurons $[7,15]$ was adopted for classification of CVLM neurons. CVLM neurons displaying an increase in firing rate evoked by noxious test stimulation were considered to be $(+)$-cells to that stimulus, while those decreasing activity were considered to be (-)-cells (Fig. 3). Neurons displaying no or only a negligible $(<10 \%)$ change in their discharge rates as a response to noxious stimulation were considered to be $(=)$ cells. Response properties of $(+)-,(-)$ - and (=)-cells of the CVLM resembled those of ON-, OFF- and NEUTRAL-cells of the RVM, respectively $[7,12]$. If a neuron could not be classified it was excluded from the study. When compared with previous studies on RVM neurons, one important difference in classification of CVLM neurons was that in the present study CVLM neurons were characterized based on their responses to noxious stimuli, independent of reflex activity, whereas reflex activ- ity is a key parameter in classification of RVM cells according to the classification scheme developed by Fields et al. [7].

Characterization of the response properties of CVLM cells consisted of the following assessments performed successively: (i) Spontaneous activity; (ii) Response to heating of the hind paw that was ipsilateral to the inflamed knee in arthritic animals. Heat stimulation consisted of a heat ramp rising at the rate of $10^{\circ} \mathrm{C} / \mathrm{s}$ from the baseline temperature of $35^{\circ} \mathrm{C}$ to the peak temperature of $54^{\circ} \mathrm{C}$ and peak duration of $10 \mathrm{~s}$. A feedback-controlled Peltier device (LTS-3 Stimulator, Thermal Devices Inc., Golden Valley, MN, USA [39]) was used for producing heat stimuli; (iii) Response to pinching of the tail for $5 \mathrm{~s}$ by a surgical clamp that produced painful sensation when applied to the hand of the experimenter; (iv) Response to colorectal distension at a noxious intensity $(80 \mathrm{mmHg}$ [29] and duration of $10 \mathrm{~s})$. Colorectal distension was produced by inflating with air a $7-8 \mathrm{~cm}$ flexible latex balloon inserted transanally into the descending colon and rectum; the pressure in the balloon was controlled by an electronic device [1]; (v) Response to cold stimulation of the plantar skin of the hind paw that was ipsilateral to the inflamed knee joint in arthritic animals. Cold stimulation consisted of a thermal ramp decreasing at the rate of $4{ }^{\circ} \mathrm{C} / \mathrm{s}$ from the baseline temperature of $35^{\circ} \mathrm{C}$ to the minimum temperature of $4{ }^{\circ} \mathrm{C}$ and with the duration of $10 \mathrm{~s}$. LTS-3 Stimulator (Thermal Devices Inc.) was used for delivering cold stimuli.

When analyzing responses of CVLM neurons to peripheral stimulation, the baseline discharge frequency recorded during a corresponding period just before the stimulation was subtracted from the discharge frequency determined during stimulation; i.e., positive values represent excitatory responses evoked by peripheral stimulation and negative ones inhibitory responses. However, when comparing the peripheral stimulus-evoked response magnitudes in cells giving an excitatory versus inhibitory response, the absolute values of responses were used for cells with an inhibitory response; i.e., when comparing peripherally evoked response magnitudes, inhibitory responses had values $>0 \mathrm{~Hz}$, instead of $<0 \mathrm{~Hz}$.

After determining the responses of a CVLM neuron to peripheral stimulation, the phasic modulation of the discharge rate of CVLM neurons by PVN neurons was assessed by microinjecting glutamate $(50 \mathrm{nmol}$ in $0.5 \mu \mathrm{L})$ into the PVN using methods described below. The discharge rate of the CVLM cells was followed up to $5 \mathrm{~min}$ after the injection of glutamate. All results from the glutamate administration were plotted against the values obtained for the same time points after saline injection in the PVN.

\subsection{Induction of arthritis}

The induction of arthritis in the knee joint was performed 7-9 days before the actual experiments, as described in detail elsewhere [2]. Briefly, 3\% kaolin and 3\% carrageenan (Sigma-Aldrich) were dissolved in distilled water and injected into the synovial cavity of the left knee joint at a volume of $0.1 \mathrm{~mL}$. This model produces mechanical hyperalgesia, which begins just in a few hours after surgery and extends up to 8 weeks [32]. In each animal, development of arthritis was verified 1-2 $\mathrm{h}$ prior to each experiment. Only those rats that vocalized every time after five flexion-extension movements of the knee joint were considered to have arthritis, and they were included in the arthritis group. A control group was injected with $0.1 \mathrm{~mL}$ of saline in the synovial cavity of the left knee joint. Control animals did not
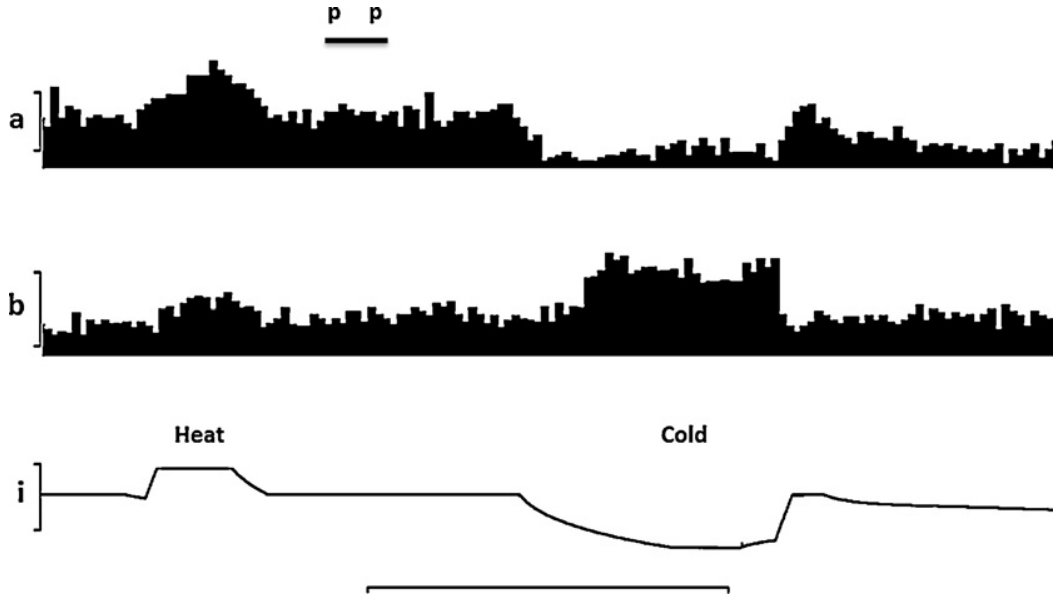

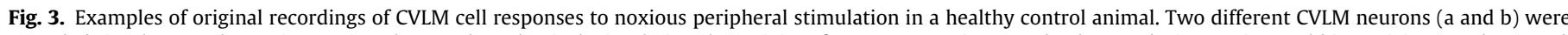

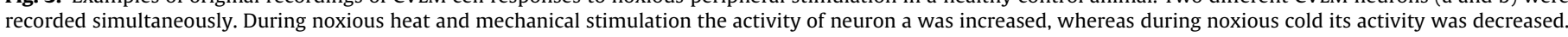

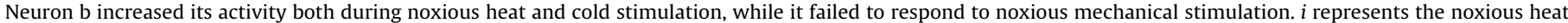

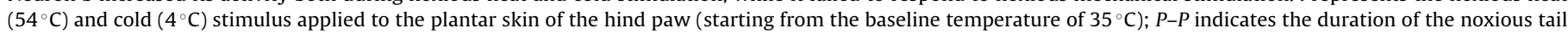
pinch (mechanical stimulation)]. The vertical calibration bar for neuronal response represents $10 \mathrm{~Hz}$ and the horizontal calibration bar represents $40 \mathrm{~s}$. 
Table 1

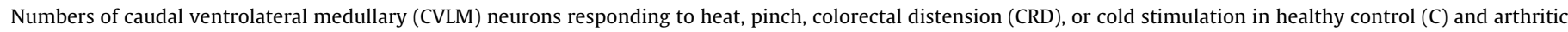

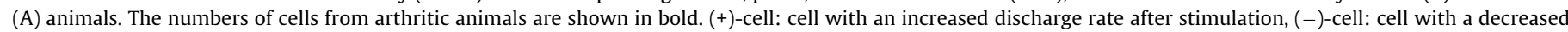
discharge rate after stimulation, (=)-cell: cell with no change in discharge rate after stimulation.

\begin{tabular}{|c|c|c|c|c|c|c|c|c|c|c|c|}
\hline \multicolumn{3}{|c|}{ Response to heat } & \multicolumn{3}{|c|}{ Response to pinch } & \multicolumn{3}{|c|}{ Response to CRD } & \multicolumn{3}{|c|}{ Response to cold } \\
\hline & $\mathrm{C}$ & A & & $\mathrm{C}$ & A & & $\mathrm{C}$ & A & & $\mathrm{C}$ & A \\
\hline \multirow[t]{3}{*}{$(+)$ cells } & 44 & 48 & $(+)$ cells & 21 & 18 & $(+)$ cells & 9 & 12 & $(+)$ cells & 14 & 15 \\
\hline & & & $(-)$ cells & 9 & 11 & $(-)$ cells & 11 & 13 & $(-)$ cells & 13 & 23 \\
\hline & & & (=) cells & 14 & 19 & $(=)$ cells & 24 & 23 & $(=)$ cells & 17 & 10 \\
\hline \multirow[t]{3}{*}{$(-)$ cells } & 17 & 19 & $(+)$ cells & 9 & 11 & $(+)$ cells & 6 & 2 & $(+)$ cells & 2 & 2 \\
\hline & & & $(-)$ cells & 6 & 4 & $(-)$ cells & 8 & 7 & $(-)$ cells & 13 & 10 \\
\hline & & & (=) cells & 2 & 4 & $(=)$ cells & 3 & 10 & (=) cells & 2 & 7 \\
\hline \multirow[t]{3}{*}{$(=)$ cells } & 17 & 12 & $(+)$ cells & 5 & 2 & $(+)$ cells & 3 & 0 & $(+)$ cells & 4 & 3 \\
\hline & & & $(-)$ cells & 0 & 4 & $(-)$ cells & 2 & 3 & $(-)$ cells & 4 & 7 \\
\hline & & & (=) cells & 12 & 6 & $(=)$ cells & 12 & 9 & $(=)$ cells & 9 & 2 \\
\hline
\end{tabular}

vocalize to any of the five consecutive flexion-extension movements of the knee joint.

\subsection{Course of the electrophysiological study}

Electrophysiological recordings of CVLM neurons were performed under pentobarbitone anesthesia one to two weeks following the induction of arthritis (or sham treatment) and at least one week following the insertion of the guide cannula for PVN injections. In CVLM recordings, the response properties of the neurons were assessed by determining spontaneous activity and the response to noxious heating of the hind paw skin, tail pinch, colorectal distension and noxious cold stimulation of the hind paw skin. The site of heat and cold stimulation was on the plantar skin of the hind paw ipsilateral both to the recording electrode in the CVLM and the arthritic knee joint. The intervals between different types of test stimulations were about $20 \mathrm{~s}$. The first testing session was the vehicle control session for the PVN. Then, after a pause of at least one min glutamate was microinjected into the PVN and testing procedure was repeated. The testing procedure was completed within 2 min, which was considered to be within the time that glutamate has its main effect and thus allowing assessing glutamate-induced changes in responses. Search for the next neuron to be studied started about $30 \mathrm{~min}$ after the testing of the previous one was completed. At the end of the recording session, electrolytic lesions were made in the recording sites, the animals were given a lethal dose of pentobarbitone and the brains were removed for histological verification of the recording and injection sites.

While it might be argued that the order and short interval of testing different submodalities of noxious stimulation or the order of testing glutamate after vehicle may have influenced the results, it should be noted that the same procedure was used for testing control and arthritic animals, and for testing different types of neurons. Therefore, differences in results between control and arthritic animals or in results between different types of cells represent arthritis-induced changes and/or cell type-associated differences rather than artificial changes induced by testing procedures or the order of testing.

\subsection{Statistics}

The differences in the number of the different types of CVLM neurons between experimental groups were analyzed using the chi-square test. Differences in neuronal discharge rates between experimental groups were assessed using $t$-test (comparisons between two groups) or two-way analysis of variance (ANOVA) followed by $t$-test with a Bonferroni correction for multiple comparisons (comparisons between 3 or more groups). A value of $P<0.05$ was considered to represent a significant difference.

\section{Results}

\subsection{Cell types in the CVLM}

CVLM neurons typically had large receptive fields covering the lower body half that included the sites of quantitative test stimulation in the plantar skin of the hind paw and the tail. CVLM cells were first classified based on their responses to noxious heat stimulation (Table 1). Neurons were classified into three types based on their responses to noxious heat stimulation of the hind paw (Fig. 4A). A group of cells was clearly excited after noxious heat stimulation and they were classified as (+)-cells. The firing rate of the second group of neurons was clearly decreased by noxious heat stimulation. These neurons were classified as (-)-cells. Finally, discharge rates in the third group of neurons were not changed by noxious heat. These neurons were classified as (=)-cells. The appli- cation of other submodalities of noxious test stimulation (noxious cold, pinch and colorectal distension) also produced an increase, decrease or failure to influence the discharge rate of the CVLM cell allowing submodality-related classification of CVLM neurons into (+)-, (-)-cells and (=)-cells (Fig. 4B-D; Table 1). It should be noted, however, that classification of the CVLM cell into a subgroup could vary with the submodality of noxious test stimulation; i.e., a CVLM cell classified as (+)-cell based on its response to heat, could be (-)cell based on its response to noxious cold and (=)-cell based on its response to noxious colorectal distension. Of the 78 CVLM neurons studied in healthy control animals, 24 had the same classification, independent of the submodality of noxious test stimulation. Number of neurons giving a similar type of response to at least three submodalities of noxious stimulation was 34 out of 78 neurons in controls. There was no obvious relationship between the recording site within the CVLM and the response type of the CVLM neuron.

\subsection{Influence of arthritis on the distribution of cell types in the CVLM}

To assess whether arthritis induces changes in the distribution of CVLM cell types or in their responses, a group of animals was tested following induction of arthritis in the knee joint. The total number of CVLM neurons studied in arthritic animals was 79 (Table 1$)$. In the CVLM of arthritic animals, the distributions of (+)-, $(-)$ - and (=)-cells, as revealed by the chi-square test, were not significantly different from the corresponding distributions in healthy control animals, independent whether the classification was based on the response to noxious heat (Fig. 4A), pinch (Fig. 4B), colorectal distension (Fig. 4C) or noxious cold (Fig. 4D).

\subsection{Spontaneous discharge rate of CVLM neurons and an attempt to modulate it by glutamatergic stimulation of the PVN}

Noxious heat-based classification of CVLM cells was used when analyzing whether spontaneous discharge rates of neurons vary with the response type of the CVLM cell or pathophysiological condition (arthritis versus controls). Cell type was a significant factor in determining the mean spontaneous discharge rate of CVLM neurons $\left(F_{2,238}=7.17, P=0.0010 ;\right.$ Fig. 5$)$. The influence of the cell type on the spontaneous discharge rate varied with the pathophysiological condition (arthritis versus controls: $F_{2,238}=5.23, P=0.0060$ ). Post hoc testing indicated that CVLM cells with the (+)-type response to noxious heat had significantly higher spontaneous discharge rates than (+)-cells in control animals, or (-)- and (=)-cells in the CVLM of arthritic animals (Fig. 5).

Influence of glutamatergic stimulation of the PVN on the spontaneous discharge rate of CVLM neurons was studied in a total of 47 CVLM neurons giving either excitatory or inhibitory response to heat; i.e., only neurons classified as (+)- or (-)-cells, respectively, 

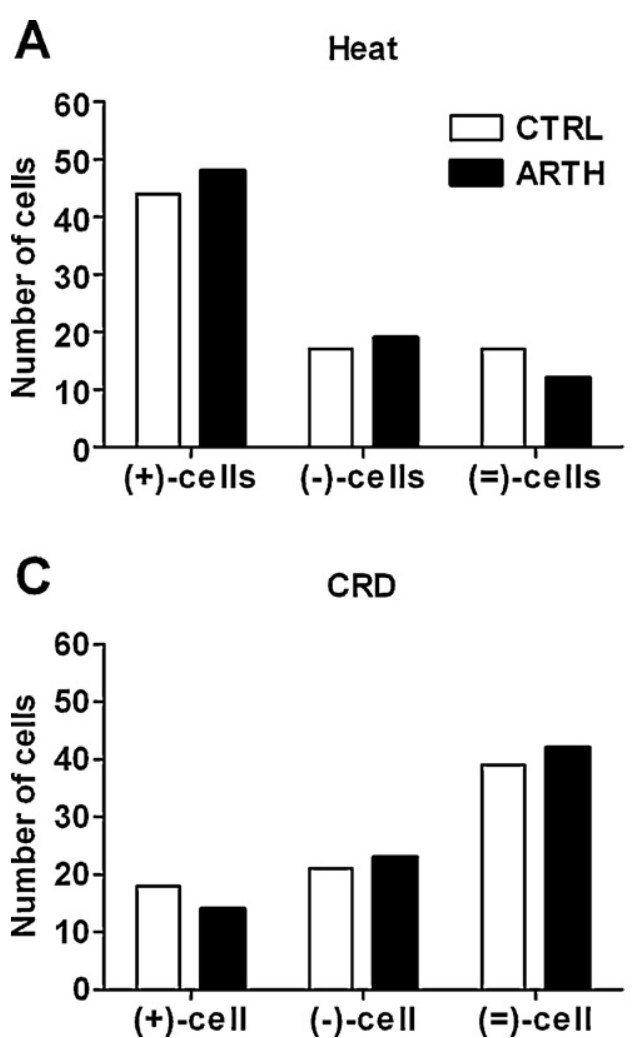

B

Pinch

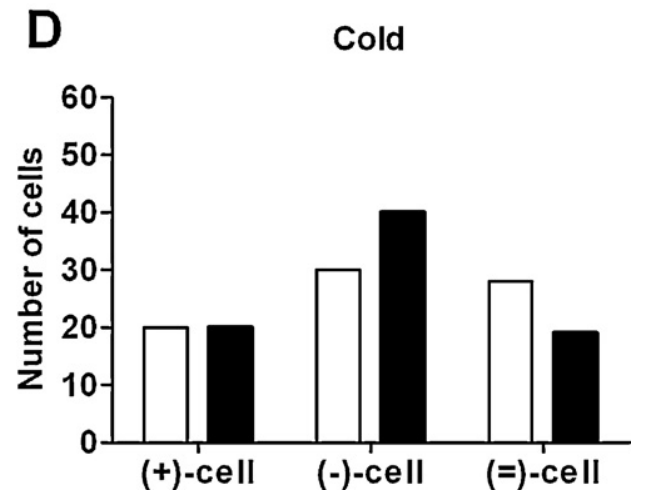

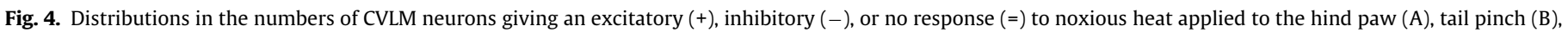
colorectal distension (CRD; C), or cold applied to the hind paw (D). CTRL: sham controls; ARTH: animals with monoarthritis of the knee joint.

based on their response to heat were studied. Of these 47 neurons, 28 were studied in control animals [21 (+)-cells and $7(-)$-cells] and 19 neurons were studied in arthritic animals [10(+)-cells and 9(-)cells]. Glutamate in the PVN failed to influence the spontaneous discharge rates of CVLM neurons in control or arthritic animals, independent of the cell type ( $F<2$ in all comparisons; not shown).

\subsection{Magnitude of the noxious stimulation-evoked response of CVLM neurons in control and arthritic animals}

When analyzing noxious stimulus-evoked responses of CVLM neurons, each submodality of test stimulation was analyzed separately. Moreover, classification of cells into (+)- and (-)-cells was

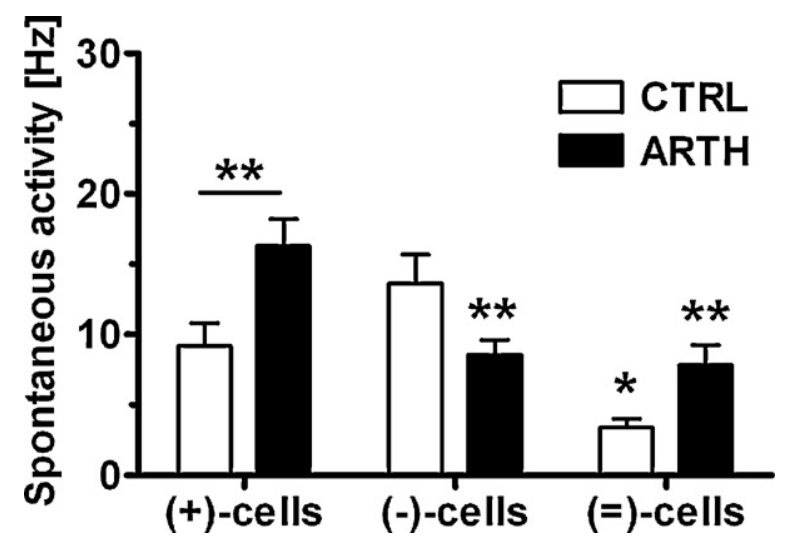

Fig. 5. Spontaneous discharge rates of CVLM neurons giving excitatory $(+)$, inhibitory $(-)$ or no response $(=)$ to noxious heat stimulation of the hind paw in healthy control (CTRL) and arthritic (ARTH) animals. Error bars represent S.E.M. $(n=21-66) .{ }^{*} P<0.05,{ }^{*} P<0.01$ ( $t$-test with a Bonferroni correction; reference: unless specified, the corresponding value in the (+)-cell group). done based on the response to the studied submodality. Furthermore, (=)-cells not responding to the studied submodality were not included when analyzing the magnitudes of the stimulus-evoked responses within each submodality. In general, arthritis influenced the magnitude of the noxious stimulation-evoked response in CVLM neurons, and the arthritis-induced modulatory effect varied with the cell type and the submodality of noxious test stimulation (Fig. 6). For the sake of clarity, absolute values of inhibitory (-)cell responses were used in statistical comparisons of differences in the magnitudes of the peripheral stimulus-evoked responses between (+)- and (-)-cells. In other words, responses $>0 \mathrm{~Hz}$ for (-)cells shown in Fig. 5 represent stimulus-evoked decreases in the discharge rate.

When noxious heat was used for test stimulation, the magnitude of the heat evoked response was significantly influenced by arthritis $\left(F_{1,62}=8.58, P=0.0047\right)$ and the cell type $\left(F_{1,62}=111.3\right.$, $P<0.0001)$. Interestingly, the influence of arthritis on the magnitude of the heat-evoked response varied with the cell type $\left(F_{1,62}=60.39, P<0.0001\right)$. In CVLM $(+)$-cells giving an excitatory response to heat, the magnitude of the heat-evoked response was decreased in arthritis, whereas in (-)-cells giving an inhibitory response to heat the absolute magnitude of the heat-evoked response was increased (Fig. $6 \mathrm{~A}$ ).

Magnitude of the noxious tail pinch-evoked response was significantly influenced by arthritis $\left(F_{1,70}=74.96, P<0.0001\right)$ and the type of CVLM cell $\left(F_{1,70}=109.7, P<0.0001\right)$. The influence of arthritis varied depending whether the cell gave an excitatory or inhibitory response to pinch $\left(F_{1,70}=36.55, P<0.0001\right)$ : CVLM cells classified as (+)-cells to pinch had an increased response to pinch in arthritic animals, whereas arthritis failed to influence the magnitude of the pinch-evoked response in (-)-cells giving an inhibitory response to pinch (Fig. 6B).

Magnitude of the noxious colorectal distension-induced response was significantly decreased in arthritis $\left(F_{1,79}=30.2\right.$, 

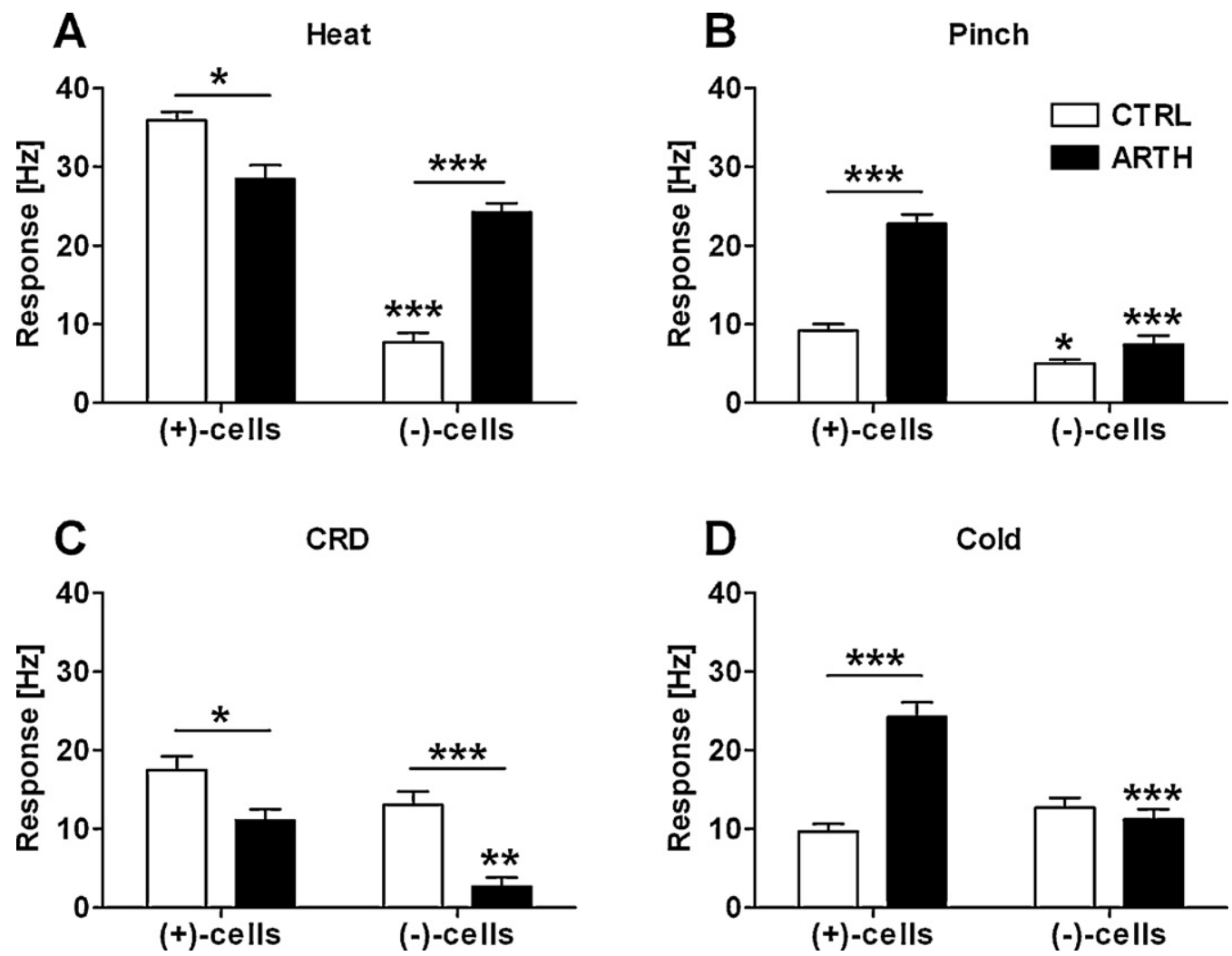

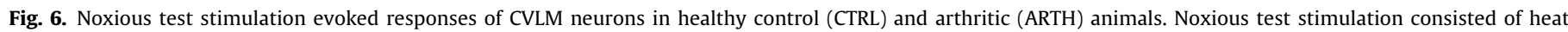

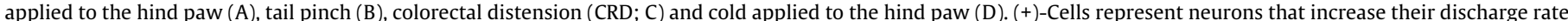

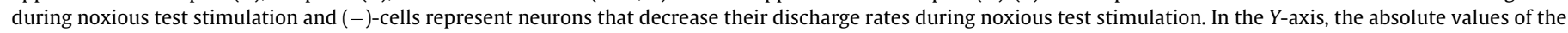

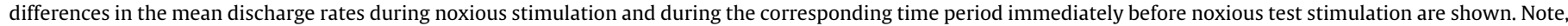

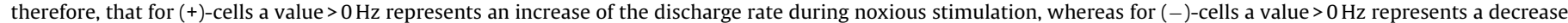

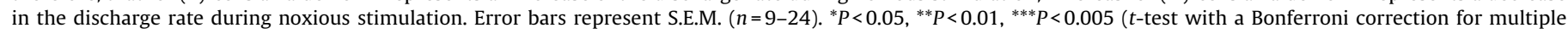
comparisons; reference: unless specified, the corresponding (+)-cell response).

$P<0.0001$; Fig. 6C). The magnitude of the colorectal distensioninduced response was significantly weaker in (-)-cells giving an inhibitory response to colorectal distension than in (+)-cells giving an excitatory response $\left(F_{1,79}=17.94, P<0.0001\right)$, independent of arthritis $\left(F_{1,79}=1.75\right.$; Fig. $\left.6 C\right)$.

Arthritis had a significant effect on the magnitude of the noxious cold-evoked response $\left(F_{1,71}=22.76, P<0.0001\right.$; Fig. $\left.6 \mathrm{D}\right)$. The arthritis-induced modulation of the cold-evoked response varied with the cell type $\left(F_{1,71}=33.93, P<0.0001\right)$ : arthritis induced a significant facilitation of the noxious cold-evoked response only in (+)-cells giving an excitatory response to cold (Fig. 6D).

\subsection{Influence by glutamatergic stimulation of the PVN on the noxious stimulus-evoked discharge rates of CVLM neurons}

To assess whether phasic activation of the PVN influences noxious stimulus-evoked responses of CVLM neurons, the magnitude of the noxious stimulus-evoked response in the CVLM neurons was determined with microinjection of glutamate/vehicle control in the PVN of control and arthritic animals. Responses to each submodality of noxious test stimulation were assessed separately, and only neurons giving an excitatory or inhibitory response to test stimulation were included in this study. For statistical analyses and illustrations of Fig. 7, the peripheral stimulus-evoked responses obtained following vehicle administration in the PVN were subtracted from the corresponding response obtained following glutamate administration in the PVN; independent of the cell type, values $<0$ represent a glutamate-induced decrease in the magnitude of the response evoked by noxious peripheral stimulation. In general, glutamate in the PVN of arthritic animals tended to suppress noxious stimulation-induced excitatory responses of CVLM neurons.

Glutamate in the PVN had a significantly different effect on the magnitude of the heat-evoked responses in (+)-cells as in $(-)$-cells $\left(F_{1,71}=13.44, P<0.0001\right.$; Fig. $\left.7 \mathrm{~A}\right)$. The heat-evoked excitatory responses, unlike inhibitory responses, were reduced by glutamatergic stimulation of the PVN. Post hoc testing indicated that the reduction of the heat-evoked excitatory response was significantly stronger in arthritic than control animals, whereas no glutamate-induced difference was observed in heatevoked inhibitory responses between control and arthritic animals (Fig. 7A).

Descending modulation of the noxious pinch-evoked response by glutamatergic stimulation of the PVN was significantly different between the CVLM cells giving an excitatory and inhibitory response to pinch $\left(F_{1,28}=5.08, P=0.032\right.$; Fig. $\left.6 \mathrm{~B}\right)$, and the difference varied with the pathophysiological condition (arthritis versus controls: $F_{1,28}=7.16, P=0.012$ ). In arthritic but not control animals, the magnitude of the pinch-evoked excitatory response in (+)-cells tended to be decreased (Fig. 7B).

Glutamatergic stimulation of the PVN had a significantly different effect on the magnitude of the colorectal distensioninduced response in CVLM neurons giving an excitatory and inhibitory response $\left(F_{1,37}=16.62, P=0.0002\right)$, and this descending modulatory effect varied with the pathophysiological condition $\left(F_{1,37}=5.38, P=0.026\right.$; Fig. $\left.7 C\right)$. Glutamate in the PVN suppressed the colorectal distension-induced excitatory but not inhibitory response of CVLM neurons, and the suppression of the excitatory response was significantly stronger in arthritic animals (Fig. 7C). 
A

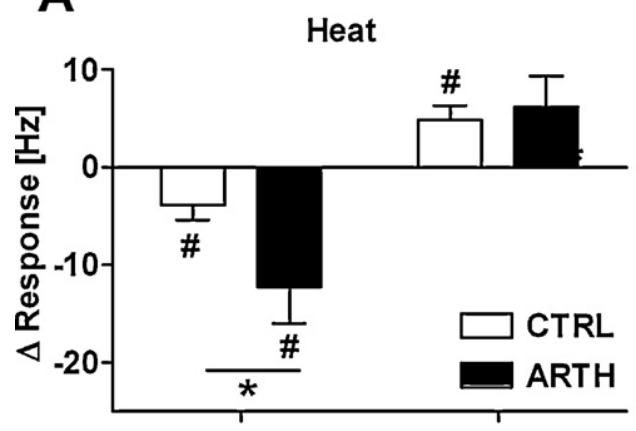

(+)-cells

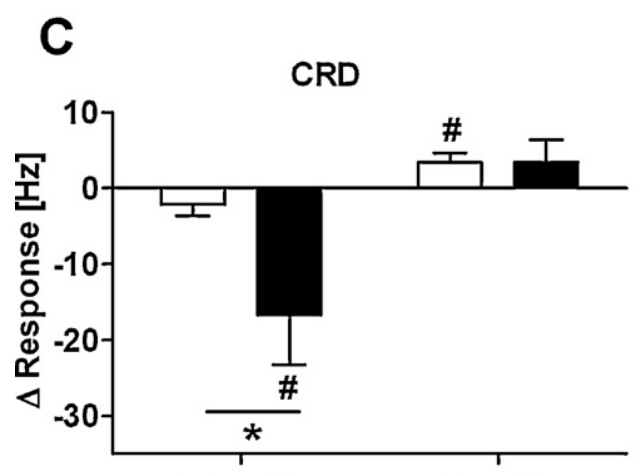

(+)-cells

(-)-cells

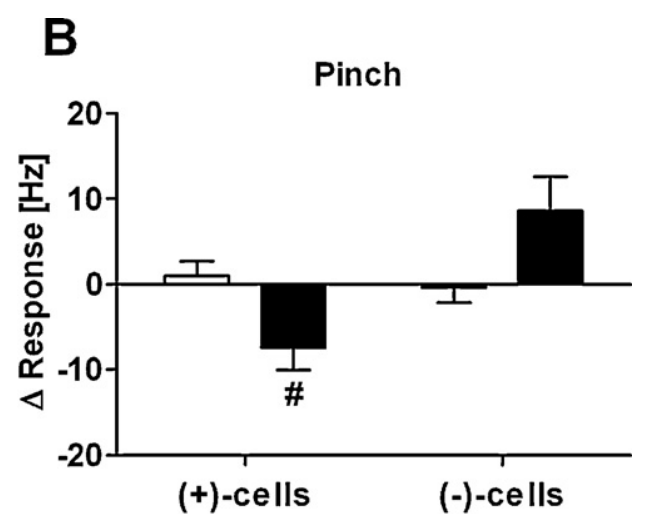

D

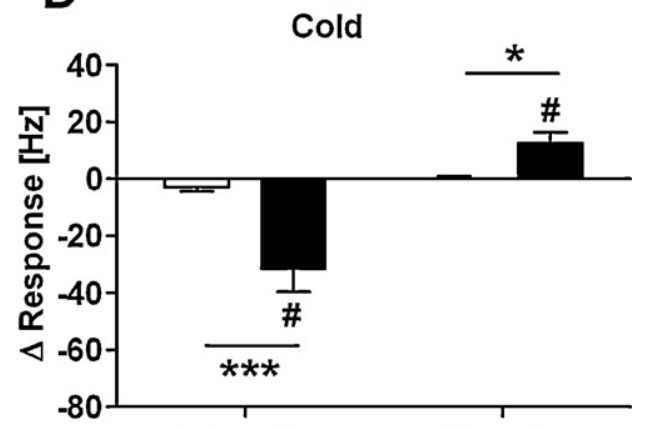

(+)-cells

(-)-cells

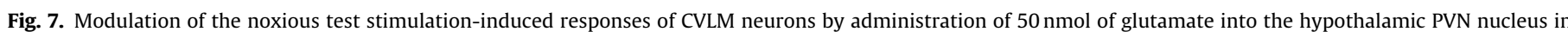

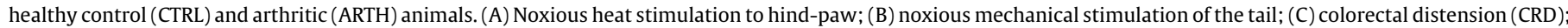

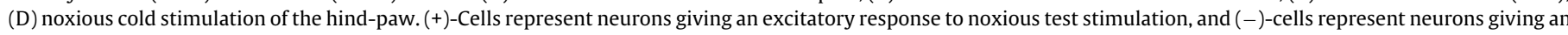

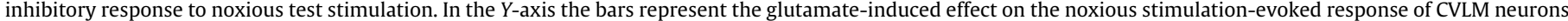

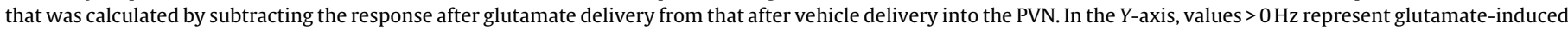

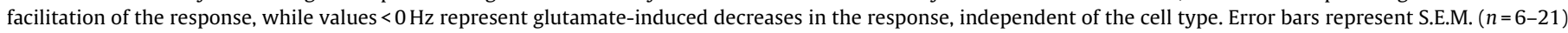

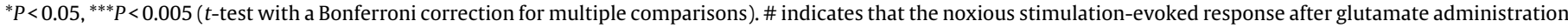
into the PVN is outside of the $95 \%$ confidence limits of the corresponding response after vehicle administration.

Noxious cold-induced response of CVLM neurons was significantly modulated by glutamatergic stimulation of the PVN. The modulatory effect was different between CVLM cells giving an excitatory versus inhibitory response to cold $\left(F_{1,37}=16.62, P=0.0002\right)$ and it varied with the pathophysiological condition $\left(F_{1,37}=5.38\right.$, $P=0.026$; Fig. 7D). The cold-evoked excitatory response in $(+)-$ cells was decreased and the inhibitory response in (-)-cells was increased by glutamate in the PVN. Both of these glutamateinduced changes were significantly stronger in arthritic than control conditions (Fig. 7D).

\section{Discussion}

The present results indicate that most of the CVLM neurons respond to noxious test stimulation and undergo changes in their firing patterns after development of arthritis. Moreover, glutamatergic activation of a hypothalamic nucleus involved in pain regulation, the PVN, was shown to reduce excitatory responses of nociceptive neurons in the CVLM of arthritic animals at a dose that failed to influence responses of CVLM neurons in controls.

\subsection{Activity patterns of CVLM neurons}

Based on their responses to noxious test stimulation, CVLM neurons could be subdivided in three groups: (i) one group of CVLM neurons was excited by noxious test stimulation and they were classified as (+)-cells; (ii) a second group of CVLM neurons gave an inhibitory response to noxious test stimulation and they were classified as (-)-cells; (iii) a third group of CVLM neurons that was classified as (=)-cells had a low basal activity which was not changed by noxious test stimulation. Previously, Fields et al. have classified RVM neurons into ON-cells that fire just prior to a reflex response induced by a noxious test stimulus, OFF-cells that stop firing just prior to a flexion reflex induced by a noxious test stimulus, and NEUTRAL-cells the firing of which is not associated with the spinal reflex response or noxious test stimulation $[7,8]$. There is an apparent similarity in response properties of CVLM (+)-, (-)-, and (=)-cells with the RVM ON-, OFF, and NEUTRAL-cells, respectively. In addition to the RVM, similar response patterns have been described also in other brainstem nuclei that are involved in regulation of nociception, such as the periaqueductal gray [14]. It should, however, be pointed out that classification of CVLM neurons in the present study was based on neuronal responses to noxious stimulation, without assessment of accompanying spinal reflex responses, unlike in the classification scheme of Fields et al. [7,15].

RVM ON-cells presumably have a pronociceptive role, whereas RVM OFF-cells are considered to contribute to antinociception $[7,8]$. While conclusive statements on the functional roles of CVLM $(+)-$ and (-)-cells still require further studies, such as parallel assessments of behaviour and neuronal activity, it is tempting to speculate that CVLM (+)-cells resembling RVM ON-cells promote nociception and CVLM (-)-cells resembling RVM OFF-cells promote antinociception. In line with this proposal, the spontaneous discharge rate of CVLM (+)-cells was increased and that of $(-)$-cells decreased in animals with a chronic pain condition, arthritis. 


\subsection{Changes in noxious stimulation-induced responses of CVLM neurons following development of arthritis}

It is well established that arthritis induces peripheral sensitization at the knee and central sensitization at the spinal cord [34] The arthritis-induced increase in impulse traffic ascending from the spinal cord could explain the increase in spontaneous activity of CVLM neurons. When considering arthritis-induced changes in neuronal responses to noxious test stimulation, it should be noted that test stimuli were applied either to the hind paw distal to the arthritic knee joint (heat and cold), to the tail (pinch) or to the viscera (colorectal distension). Therefore, we cannot exclude possible regionally restricted changes in responses to stimulation of the inflamed tissue in the knee. The results allow concluding that the arthritis-induced changes in the noxious test stimulation-evoked responses of CVLM neurons reflect net effects of complex central interactions that may vary from central summation to inhibition of afferent barrages from the test stimulus site and the inflamed joint.

The noxious tail pinch-evoked excitatory response of CVLM $(+)$-cells was facilitated following development of arthritis of the knee, while the pinch-evoked inhibitory response in CVLM neurons was not changed. Similarly, the noxious cold-evoked excitatory response was enhanced in arthritis, whereas the cold-evoked inhibitory response was not changed. In contrast, the excitatory response of CVLM neurons to noxious heat-stimulation of the plantar skin of the hind paw was decreased in arthritis of the knee joint and this was accompanied by an increased inhibitory response to heat. It remains to be studied whether these neuronal changes in the CVLM are associated with corresponding behavioural changes (increased mechanical and cold responses and decreased heat sensitivity outside of the inflamed joint) as expected if CVLM (+)-cells promote and (-)-cells suppress nociception. With noxious visceral stimulation, the interpretations from arthritis-induced changes in response properties of CVLM neurons to potential behavioural changes are complicated by the finding that the changes of response properties in CVLM neurons (decreased excitatory and inhibitory responses to colorectal distension) may have opposed each other's effects. The arthritis-induced facilitation of the pinch-evoked excitatory responses in CVLM neurons is in line with abundant evidence indicating that hypersensitivity to mechanical stimulation is characteristic for arthritis [34]. However, functional significance of the arthritis-induced changes in the responses of CVLM neurons to cold, heat and colorectal distension, if any, still remains to be studied.

\subsection{Influence of the PVN on neuronal activity in the CVLM}

In the present study, the noxious stimulation-induced excitatory responses of CVLM (+)-cells were reduced in arthritic but not control animals following administration of glutamate into the PVN at a dose that has proved effective in producing spinal antinociception both in healthy control and arthritic animals [31]. Since the PVN-induced suppression of nociceptive responses was not associated with a suppression of spontaneous discharge rates of CVLM neurons, the PVN-induced antinociceptive effect in the CVLM may be explained due to an effect presynaptic to the CVLM rather than a direct action on the CVLM neurons. In other words, the PVNinduced antinociceptive effect in the CVLM may, at least partly, be secondary to the direct PVN-induced descending influence upon the spinal cord [4-6]. Independent of the route of action from the PVN to the CVLM, the inhibition of excitatory responses of CVLM $(+)$-cells by glutamate injection into the PVN at an antinociceptive dose supports the proposal that CVLM (+)-cells have a pronociceptive role.

\subsection{Submodality-related variability in classification}

The present classification of CVLM neurons was performed based on responses to four submodalities of noxious test stimulation (pinch, heat, cold and colorectal distension). In many CVLM neurons, classification varied depending on the submodality of noxious test stimulation. For example, a cell giving an excitatory response to heat could give an inhibitory response to pinch. Only in a minority of the CVLM cells the classification was the same, independent of the submodality of test stimulation. Excitatory responses of CVLM neurons were most commonly observed with heat, whereas visceral stimulation most commonly failed to produce a response. With noxious cold, the CVLM neurons most commonly failed to give a response or gave an inhibitory response. Earlier studies have shown that classification of RVM neurons may vary with the submodality of test stimulation [35] indicating that the submodality-dependent variation in the neuronal response type may not be an uncommon feature in pain-regulatory brainstem nuclei. In the RVM, neurons with a submodality-dependent variation in their response properties have been called atypical RVM neurons [35]. Earlier studies indicate that inflammatory conditions may induce a switch of the cell phenotype in the RVM, as indicated by a decreased proportion of RVM NEUTRAL-cells [26,27]. In CVLM neurons of the present study, however, arthritis failed to induce significant changes in the distributions of neurons giving excitatory, inhibitory or no response to noxious stimulation.

Different submodalities of noxious stimulation activate at least partly different populations of primary afferent nerve fibers, from which signals are carried to the brain via multiple ascending pathways [19]. The finding that the response evoked by the same submodality of noxious stimulation varied from excitation to inhibition among CVLM cells indicates that even within the same submodality of noxious stimulation, the evoked response is carried to the CVLM via at least partly different circuitries. The diversity in afferent pathways probably explains the heterogeneous response properties of CVLM neurons. Among functional explanations for the apparent complexity of the present findings is that the efferent projection, which was not characterized in the present study, determines the response properties of the CVLM neuron and its role in pain regulation. Alternatively, the net effect induced by various subtypes of the CVLM neurons is critical for triggering the CVLM-induced effect on pain processing. Further studies are still needed to determine whether a labelled-line efferent mechanism or a population code of diverse responses is a more likely explanation for the CVLM-induced action on pain processing.

\section{Conclusions}

There is substantial evidence indicating that the CVLM is involved in regulation of pain [38], which, depending on the neurotransmitter system and pathophysiological condition, may vary from pain facilitation $[22,24]$ to inhibition $[9-11,13,21]$. The present results extend these earlier findings by demonstrating the presence of CVLM cells that increase [(+)-cells] or decrease [(-)cells] their responses following peripheral noxious stimulation and change their responses properties after development of a chronic pain condition, arthritis. Moreover, activation of the hypothalamic PVN that has been associated with descending pain inhibition reduced excitatory responses of nociceptive CVLM in arthritis, which effect, however, may be secondary to a direct antinociceptive effect of the PVN upon the spinal cord [4-6]. The responses properties of CVLM neurons are in line with the concept that the CVLM has a role in processing of nociceptive signals in arthritic as well as healthy control animals. 


\section{Conflict of interests}

The authors declare that they have no conflict of interests.

\section{Acknowledgements}

This study was supported by grants from the Academy of Finland, the Center for International Mobility (CIMO) and the Sigrid Jusélius Foundation, Helsinki, Finland, and the Grünenthal Foundation, the FCT Project no. PTDC/SAU-NEU/108557/2008 from Science and Technology Foundation (FCT) and FEDER, Lisbon, Portugal.

\section{References}

[1] R.H. Anderson, T.J. Ness, G.F. Gebhart, A distension control device useful for quantitative studies of hollow organ sensation, Physiol. Behav. 41 (1987) 635-638.

[2] O.B. Ansah, A. Pertovaara, Peripheral suppression of arthritic pain by intraarticular fadolmidine, an $\alpha_{2}$-adrenoceptor agonist, in the rat, Anesth. Analg. 105 (2007) 245-250.

[3] A. Armario, The hypothalamic-pituitary-adrenal axis: what can it tell us about stressors? CNS Neurol. Disord. Drug Targets 5 (2006) 485-501.

[4] M. Condés-Lara, G. Rojas-Piloni, G. Martínez-Lorenzana, J. RodríguezJiménez, M. Lopéz-Hidalgo, M.J. Freund-Mercier, Paraventricular hypothalamic influences on spinal nociceptive processing, Brain Res. 1081 (2006) 126-137.

[5] M. Condés-Lara, G. Rojas-Piloni, G. Martínez-Lorenzana, J. Rodríguez-Jiménez, Paraventricular hypothalamic oxytocinergic cells responding to noxious stimulation and projecting to the spinal dorsal horn represent a homeostatic analgesic mechanism, Eur. J. Neurosci. 30 (2009) 1056-1063.

[6] S. DelaTorre, G. Rojas-Piloni, G. Martínez-Lorenzana, J. Rodríguez-Jiménez, L. Villanueva, M. Condés-Lara, Paraventricular oxytocinergic hypothalamic prevention or interruption of long-term potentiation in dorsal horn nociceptive neurons: electrophysiological and behavioral evidence, Pain 144 (2009) 320-328.

[7] H.L. Fields, J. Bry, I. Hentall, G. Zorman, The activity of neurons in the rostral medulla of the rat during withdrawal from noxious heat, J. Neurosci. 3 (1983) 2545-2552.

[8] H.L. Fields, A.I. Basbaum, M.M. Heinricher, Central nervous system mechanisms of pain modulation, in: S.B. McMahon, M. Koltzenburg (Eds.) Wall and Melzack's Textbook of Pain, 5th ed., Elsevier, China, 2006, pp. $125-142$.

[9] F.W. Foong, A.W. Duggan, Brain-stem areas tonically inhibiting dorsal horn neurones: studies with microinjection of the GABA analogue piperidine-4sulphonic acid, Pain 27 (1986) 361-371.

[10] O. Gall, D. Bouhassira, D. Chitour, D. Le Bars, Involvement of the caudal medulla in negative feedback mechanisms triggered by spatial summation of nociceptive inputs, J. Neurophysiol. 79 (1998) 304-311.

[11] G.F. Gebhart, M.H. Ossipov, Characterization of inhibition of the spinal nociceptive tail-flick reflex in the rat from the medullary lateral reticular nucleus, J. Neurosci. 6 (1986) 701-713.

[12] L. Gonçalves, A. Almeida, A. Pertovaara, Pronociceptive changes in response properties of rostroventromedial medullary neurons in a rat model of peripheral neuropathy, Eur. J. Neurosci. 26 (2007) 2188-2195.

[13] J.G. Hall, A.W. Duggan, C.R. Morton, S.M. Johnson, The location of brainstem neurons tonically inhibiting dorsal horn neurons of the cat, Brain Res. 244 (1982) 215-222.

[14] M.M. Heinricher, Z. Cheng, H.L. Fields, Evidence for two classes of nociceptive modulating neurons in the periaqueductal gray, J. Neurosci. 7 (1987) 271-278.

[15] M.M. Heinricher, I. Tavares, J.L. Leith, B.M. Lumb, Descending control of nociception: specificity, recruitment and plasticity, Brain Res. Rev. 60 (2009) 214-225.

[16] A.J. Janss, G.F. Gebhart, Spinal monoaminergic receptors mediate the antinociception produced by glutamate in the medullary lateral reticular nucleus, J. Neurosci. 7 (1987) 2862-2873.
[17] H. Kawano, S. Masuko, Neurons in the caudal ventrolateral medulla projecting to the paraventricular hypothalamic nucleus receive synaptic inputs from the nucleus of the solitary tract: a light and electron microscopic double-labeling study in the rat, Neurosci. Lett. 218 (1996) 33-36.

[18] T.L. Krukoff, K.H. Harris, E. Linetsky, J.H. Jhamandas, Expression of c-fos protein in rat brain elicited by electrical and chemical stimulation of the hypothalamic paraventricular nucleus, Neuroendocrinology 59 (1994) 590-602.

[19] D. Le Bars, M. Gozariu, S.W. Cadden, Animal models in nociception, Pharmacol Rev. 53 (2001) 597-652.

[20] D. Lima, A. Albino-Teixeira, I. Tavares, The caudal medullary ventrolateral reticular formation in nociceptive-cardiovascular integration. An experimental study in the rat, Exp. Physiol. 8 (7) (2002) 267-274

[21] H. Mansikka, A. Pertovaara, The role of alpha-2-adrenoceptors of the medullary lateral reticular nucleus in spinal antinociception in rats, Brain Res. Bull. 37 (1995) 633-638.

[22] H. Mansikka, J.J. Idänpään-Heikkilä, A. Pertovaara, Different roles of alpha-2adrenoceptors of the medulla versus the spinal cord in modulation of mustard oil-induced central hyperalgesia in rats, Eur. J. Pharmacol. 297 (1996) 19-26.

[23] J. Marques-Lopes, M. Pinto, M. Morato, D. Patinha, A. Albino-Teixeira, I. Tavares, Microinjection of angiotensin II in the caudal ventrolateral medulla induces hyperalgesia, Neuroscience 158 (2009) 1301-1310.

[24] J. Marques-Lopes, D. Pinho, A. Albino-Teixeira, I. Tavares, The hyperalgesic effects induced by the injection of angiotensin II into the caudal ventrolatera medulla are mediated by the pontine A5 noradrenergic cell group, Brain Res. 1325 (2010) 41-52.

[25] I. Martins, L. Cabral, A. Pinto, S.P. Wilson, D. Lima, I. Tavares, Reversal of inflammatory pain by HSV-1-mediated overexpression of enkephalin in the caudal ventrolateral medulla reverses inflammatory pain, Eur. J. Pain (2011), doi:10.1016/j.ejpain.2011.04.007.

[26] K. Miki, Q.Q. Zhou, W. Guo, Y. Guan, R. Terayama, R. Dubner, K. Ren, Changes in gene expression and neuronal phenotype in brain stem pain modulatory circuitry after inflammation, J. Neurophysiol. 87 (2002) 750-760.

[27] J. Montagne-Clavel, J.L. Olivéras, Are ventromedial medulla neuronal properties modified by chronic peripheral inflammation? A single-unit study in the awake freely moving polyarthritic rat, Brain Res. 657 (1994) 92-104.

[28] R.D. Myers, Injection of solutions into cerebral tissue: relation between volume and diffusion, Physiol. Behav. 1 (1966) 171-174.

[29] T.J. Ness, A. Randich, G.F. Gebhart, Further behavioral evidence that colorectal distension is a 'noxious' visceral stimulus in rats, Neurosci. Lett. 131 (1991) 113-116.

[30] G. Paxinos, C. Watson, The Rat Brain in Stereotaxic Coordinates, Academic Press, Sydney, 2005.

[31] F. Pinto-Ribeiro, O.B. Ansah, A. Almeida, A. Pertovaara, Influence of arthritis on descending modulation of nociception from the paraventricular nucleus of the hypothalamus, Brain Res. 1197 (2008) 63-75.

[32] R. Radhakrishnan, S.A. Moore, K.A. Sluka, Unilateral carrageenan injection into muscle or joint induces chronic bilateral hyperalgesia in rats, Pain 104 (2003) 567-577.

[33] G. Rojas-Piloni, G. Martínez-Lorenzana, S. Dela Torre, M. Condés-Lara, Nociceptive spinothalamic tract and postsynaptic dorsal column neurons are modulated by paraventricular hypothalamic activation, Eur. J. Neurosci. 28 (2008) 546-558

[34] H.G. Schaible, F. Richter, A. Ebersberger, M.K. Boettger, H. Vanegas, G. Natura, E. Vazquez, G. Segond von Banchet, Joint pain, Exp. Brain Res. 196 (2009) 153-162.

[35] C. Schnell, C. Ulucan, J. Ellrich, Atypical on-, off- and neutral cells in the rostral ventromedial medulla oblongata in rat, Exp. Brain Res. 145 (2002) 64-75.

[36] I. Tavares, A. Almeida, A. Albino-Teixeira, D. Lima, Lesions of the caudal ventrolateral medulla block the hypertension-induced inhibition of noxious-evoked c-fos expression in the rat spinal cord, Eur. J. Pain 1 (1997) 149-160.

[37] I. Tavares, D. Lima, A. Coimbra, The pontine A5 noradrenergic cells which project to the spinal cord dorsal horn are reciprocally connected with the caudal ventrolateral medulla in the rat, Eur. J. Neurosci. 9 (1997) 2452-2461.

[38] I. Tavares, D. Lima, The caudal ventrolateral medulla as an important inhibitory modulator of pain transmission in the spinal cord, J. Pain 3 (2002) 337-346.

[39] G.L. Wilcox, G.J. Giesler Jr., An instrument using a multiple layer Peltier device to change skin temperature rapidly, Brain Res. Bull. 12 (1984) 143-146.

[40] R. Yirmiya, S. Ben-Eliyahu, Y. Shavit, P. Marek, J.C. Liebeskind, Stimulation of the hypothalamic paraventricular nucleus produces analgesia not mediated by vasopressin or endogenous opioids, Brain Res. 537 (1990) 169-174. 